Vol. LXIV No 12014

\title{
AUGMENTED REALITY \\ A REVIEW ON TECHNOLOGY AND APPLICATIONS
}

\author{
RADU EMANUIL PETRUSE \\ Faculty of Engineering/Department of Industrial Engineering and Management, “Lucian Blaga” University of \\ Sibiu, Romania, radu.petruse@ulbsibiu.ro \\ IOAN BONDREA \\ Faculty of Engineering/Department of Industrial Engineering and Management, "Lucian Blaga” University of \\ Sibiu, Romania, ioan.bondrea@ulbsibiu.ro
}

\begin{abstract}
We present in this paper an overview of the concepts and potential industrial Augmented Reality applications that can be very efficient. We also present the basic technological requirements for an AR system
\end{abstract}

Key words: Augmented Reality, AR applications, AR technologies

\section{Introduction}

All products used by individuals or society are the result of simple or complex manufacturing processes. To be competitive, today's organizations must be able to increase the value of products, to be able to produce high precision and quality in the shortest time and at a cost compatible with market demand. Thus, both products and manufacturing processes in the industrial field are becoming increasingly complex. Simultaneously, the time between demand and desired result is declining. In consequence, time is one of the most important economic resources, although not shown anywhere in the inventories of any company, it remains effectively a hidden resource (Brîndaşu, 2008).

In order to reduce costs and production time but also to increase the efficiency of product development, the digitalization rate of computer-assisted technology is in continuous progress (Figure 1).

Figure 1 Tendency to digitalization of technology (Xiumin Fan, 2011)

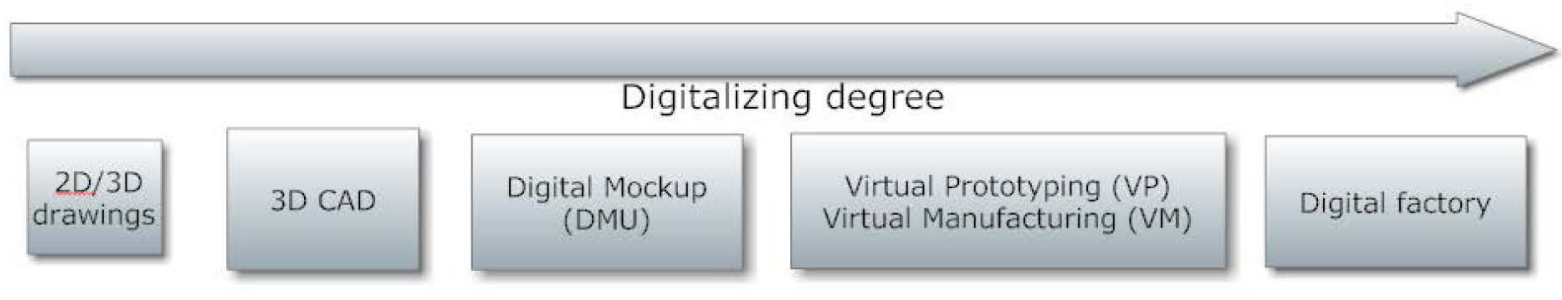

Under these circumstances, innovations regarding the manufacturing process meet extraordinary challenges.

A solution to this issue can be the brought by implementing Augmented Reality in the product's development processes.

\section{Virtual Reality and Augmented Reality}

Virtual reality (VR) and augmented reality (AR) technologies are the basis for the development of the product's virtual prototype. VR and AR form a user-friendly interface between the user and a virtual environment, environment which facilitates the exploration of a new product and its functionalities. 
VR is built of an entirely computer generated three-dimensional environment, in which the user can manipulate and interact in real time with a realistic representation of the product. AR drives the technology a step further. In contrast to VR, AR enriches the user's view of the real environment with virtual objects placed in real time and in proper position, all directly influenced by the user's perspective.

\subsection{Augmented reality}

Augmented reality is a new tool for human-computer interaction, which overlaps computer generated information (digital) on the real environment. Displaying and overlapping information is context sensitive, meaning that they depend on the observed objects. (R. Azuma, 2001)

\subsection{Brief History of Augmented Reality}

Augmented Reality is that technology which will create the next generation of user interface based on reality. Today, AR moves from research laboratories around the world into various industrial fields and markets. (D.W.F. van Krevelen, 2010)

Determining the exact moment when the concept of augmented reality was launched is difficult but one of the first examples of AR use can be traced back to August 28, 1962 when Morton Heilig patents an image, sound, vibration, and smell simulator called Sensorama.

Although it happened a few years before the invention of the personal computer, in 1970 another prototype of AR was developed by Ivan Sutherland and his students at Harvard and Utah Universities. This prototype represents the first HMD (Head Mounted Display), a helmet-shaped viewing device designed to immerse the user in a visually simulated 3D environment.

During the years 1970-1980 research in this field was done by a small group of researchers from: U.S. Air Force Armstrong Laboratory, Massachusetts Institute of Technology and the University of North Carolina. During this time devices were placed on the market such as Sony Walkman (1979), digital watches and personal digital assistants. These devices have paved the way for the invention of a portable computer which, today, has come to be a mobile platform that supports AR.

In the industrial field AR was first used experimentally by Professor Thoma P. Caudell in 1990 within the Boeing Company. AR has been adopted to improve the assembly of electrical cables in airplane bodies. But mobile AR as it is known today was inaccessible until a few years later when Steve Mann developed a GPS-based system that assisted persons with impaired sight in movement. This system overlaps audio signals on reality.

After the development of mobile computers which overlapped graphical data on the physical environment, S. Feiner introduced in 1997 a prototype of mobile AR that displayed graphical information about the university campus.

In the late 90's, AR becomes a distinct field of research, thus emerging in conferences, symposiums and even companies concerned with this technology. Also, opensource software solutions were developed, enabling the rapid construction of digital information added over reality.

In recent years the popularity of augmented reality increased, AR being adopted in various fields, from the military field to entertainment. This popularity is mainly due to rapid technological change in recent years. But AR became accessible together with the revolution of mobile phones and portable computers.

A survey conducted in 2010 by German Research Centre for Artificial Intelligence revealed a spectacular rise for AR publicity, from $56 \%$ to $85 \%$ compared to a similar survey in 2007 . Also $77.8 \%$ of the participants (industrial representatives) could envision the use of AR in their companies.

Industry analyst Tomi Ahonen recently stated that AR might be the $8^{\text {th }}$ mass market to evolve, following print, recordings, cinema, radio, TV, the Internet and mobile technologies. Also that AR audience may pass 1 billion users by 2020 (figure 2). 
Figure 2. AR's audience evolution according to Tomi Ahonen (Ahonen, 2013)

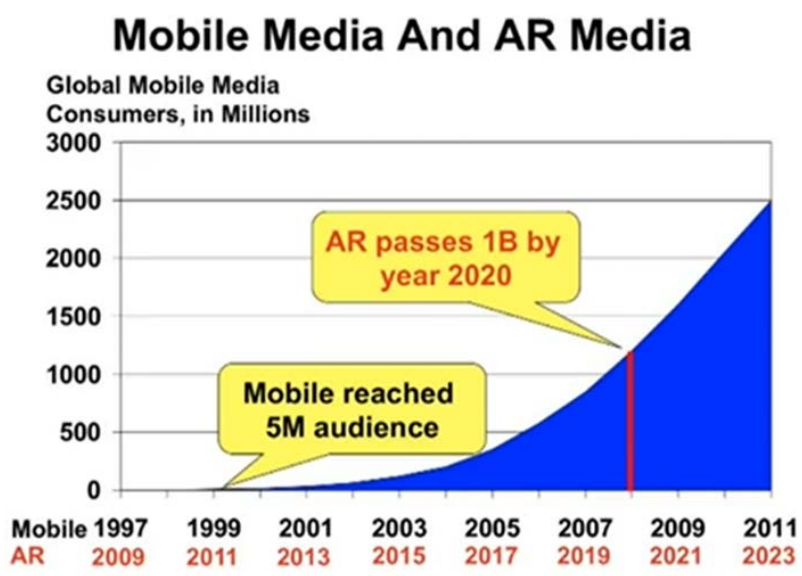

\section{AR Technological requirements}

The degree of technology required to use the AR is quite high, which contributed to the late maturation of the technology. But thanks to technology development, hardware and software resources required are not expensive. Moreover, the key components required to build a system for AR remained the same from the prototype developed by Ivan Sutherland in 1970. Display, tracking methods, computer generated graphics and AR software are essential in most experiments with AR.

\subsection{Display (display technology)}

Display represents how the user perceives augmented reality. This section channels itself on the visual display (Figure 3) although there are cases where the augmented reality uses auditory and tactile signals while the use of olfactory and gustatory senses is less developed or nonexistent. AR displays can be categorized into three divisions based on their location between the user and the physical environment: worn on the head, handheld and spatially localized (Figure 3).

Fundamentally, there are three methods to display visual AR (Figure 3). Video playback is the closest to VR. The user perceives the physical environment through a visual rendering system (monitor, display phones, etc.) that displays a "live stream" of reality upon which AR elements are superimposed.

\section{Figure 3 Technologies for augmented reality visualization}

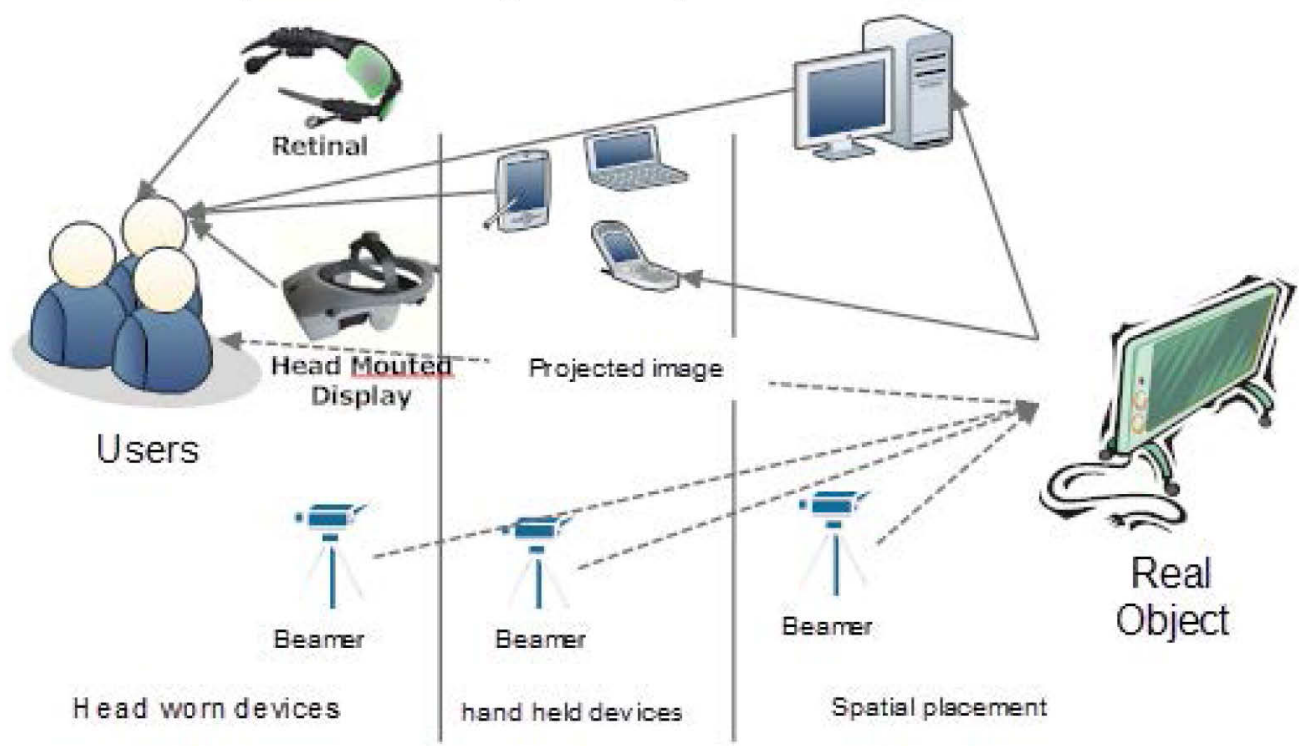

Another way to view AR, first approached by Ivan Sutherland, is the retina display system, a system that does not influence the perception of the real environment, but displays augmented reality using transparent lenses.

A third method of AR display functions by projecting AR digital information over real environment. 3D holographic projection is poorly developed but, in the upcoming years, it will certainly be widespread and affordable.

\subsection{Tracking and detection sensors}


Before an AR technology could be able to display virtual objects in a real environment, the system must be able to measure and track the user's approximate movements, preferably with six degrees of freedom.

A detected landmark in the real environment is needed, according to which the overlap of augmented reality can be correctly achieved. In addition, much of the media must be prepared before the AR technology is able to record the user's movements, although not all tracking methods work in any environment. Until this day, the determination of the user's orientation is still a complex problem for which there is no single solution.

Tracking and detection techniques depend on landmarks from the real environment, often 3D geometric models. To annotate, for example, windows, entrances or rooms, an AR technology must know their location according to the position where the user is found and his visual field.

Sometimes, these annotations can be hidden from view if the physical landmark in the environment allows it. For example, when an annotated building is only partially visible, being covered by other objects, annotation should highlight only the visible portions.

Fortunately, most parts of the environment landmarks do not require precision in terms of texture or material. Typically, a three-dimensional set of points is sufficient to provide buildings hidden from view and, in particular, to enable the user to see through the walls.

\subsection{Software}

The essential components of AR include combining real and virtual objects, 3D recording and realtime interaction. In recent years, various algorithms have been developed to address problems of recording and tracking in AR. Based on this algorithm, a number of software platforms have been transformed to facilitate the development of AR various specific applications.

Tracking and recording is the core technology that enables the implementation of AR. Without accurate tracking and registration, virtual and real objects cannot be merged. Various sensors were used to track the users' direction of view of users, essential to achieve static and dynamic entries. Main methods of AR tracking software are based on "Computer Vision" which analyses real-time video images to estimate the camera position. Methods for tracking and recording of AR systems can be classified into three categories: based on markers (marker based) based on natural features, and based on certain models (Figure 4).

\section{Figure 4 Tracking based on models}
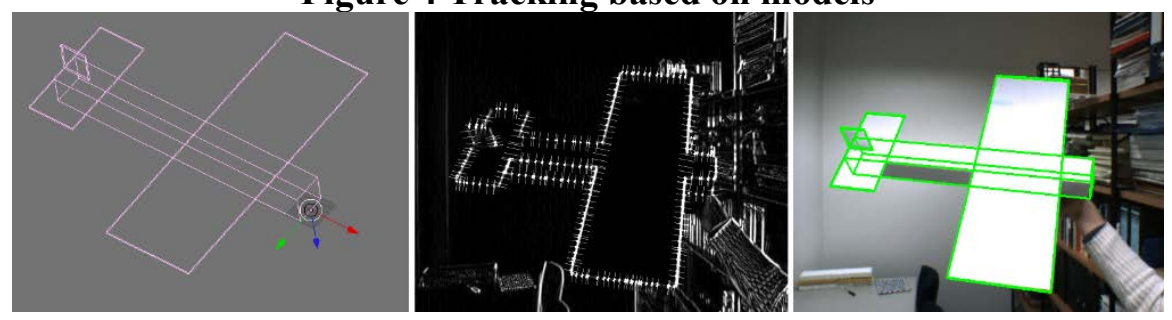

\subsection{Intellectual Content}

The commercial success of AR technologies will depend very much on the types of content available. Scientific and industrial applications are normally based on specialized content, but presenting the normal the user with commercial content will remain a challenge if AR will not come to be used in everyday life.

Creating or recording dynamic content could find help in the already existing techniques in film, games, and the computer-aided design technologies.

\section{AR application areas}

Over the years researchers and manufacturers have found an increasing number of areas where the use of augmented reality can be a benefit. The first prototypes of AR focused on the military, industrial and medical, but with technological advances AR was used for commercial purposes, entertainment and education. The latter triggered the AR prevalence and widespread use.

Maturation of AR technologies together with the hardware advances influences AR applications in becoming more diverse. This statement is strengthened by Xiangyu Wang's 2012 study on augmented reality. This study was performed on 120 AR articles between 2005 and 2011 within the normative literature to provide a state of the art of AR implications and classification. As it can be seen in figure 5 this study disclosed an increase in AR interest and also a variation expansion of applications. 
AR is currently applied in many domains such as: advertising, entertainment, education, medical purposes (image guided surgery), driving aids, military training and it can also be used for industrial applications.

Figure 5. AR articles between 2005 and 2011 and their classification (Xiangyu Wang, 2013)

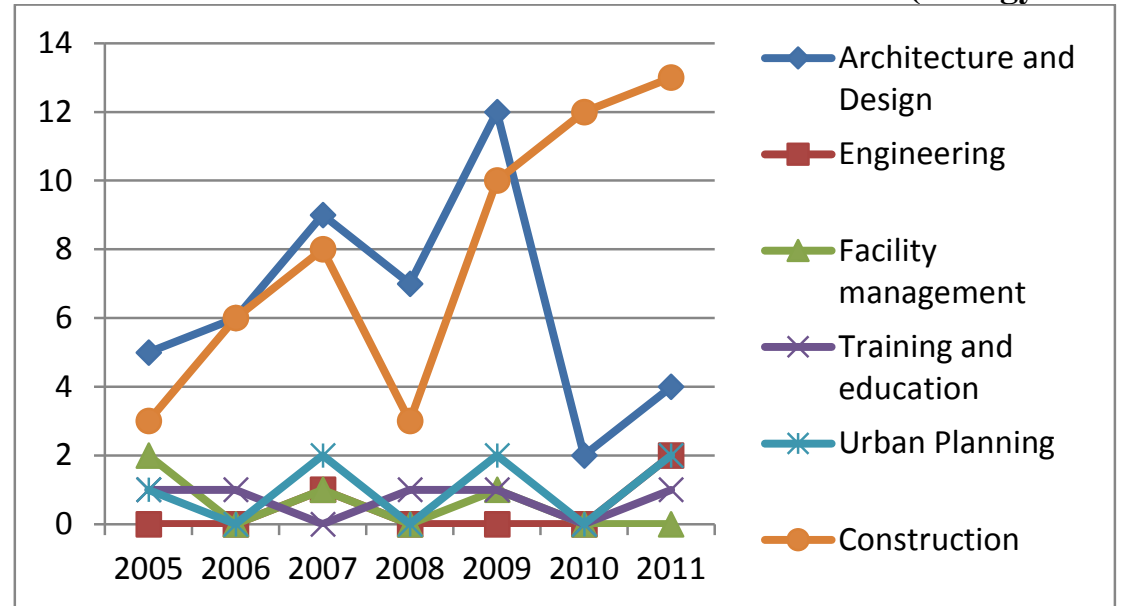

\subsection{AR used for personal use}

One of the largest markets for AR is in the range of applications for smartphones, tablets, etc. AR can become an advanced method, more natural, the interaction between the user and portable devices. For example AR can integrate communication via phone or email digital overlays that take account of context, can provide navigation guidance or offer a control interface for different devices in everyday life (DWF van Krevelen, 2010).

\subsection{AR used for entertainment}

Like virtual reality, AR can be used by the entertainment industry to create games and to increase the visibility of important issues in sports.

Video games are an important candidate for AR application, but the key problem is safety and space. The success of physically stimulating video games (Nintendo Wii) demonstrated that users want from a game more than "sitting on that couch." Thus, this area is expected to be highly developed RA in the future.

A platform that facilitates this is ARCADE (Augmented Reality Computing Arena for Digital Entertainment) (Michael R. Lyu). This platform is a software package containing tracking algorithms, design and configuration of digital objects.

\subsection{AR used in education and training}

In this field have been conducted many studies on the design of AR-based tool to improve the training process.

An application of AR in education was developed by Richard E. for children aged 7 to 11 years to test and improve decision-making ability. This application (Arve - Augmented Reality Applied to Vegetable Field Figure) allows children to manipulate entities (plants) digital 2D and 3D in an easy and intuitive. The task is to order the children's category.

\subsection{AR used in medicine}

Medical personnel can receive important information delivered directly to AR glasses. Many approaches for implementing RA medicine use image overlays generated by X-rays, ultrasounds, CT scans, etc.

The success of laparoscopy as a surgical technique that is due to the surgeon's the ability to investigate the internal cavity of the patient through small incisions. Operations carried out by so little evasive techniques reduce trauma, shortens hospitalization and allows the patient to recover faster. Although laparoscopy offers many benefits, it is difficult to use because of visual limitations. But this disadvantage can be canceled using AR. As reported by Henry Fuchs, such a system can restore the visual space of a surgeon. Taking into account the movements of the surgeon, his vision is close to that of the patient during an operation with large opening.

\subsection{AR used in the military field}

Satellite navigation systems, HMD's and many other technologies used for augmented reality are the result of research and funding from the military.

Applications of AR in other civil fields can be applied effectively in the military. Some of these applications are: support for navigation, improving communications, maintenance and emergency care. 
Other benefits of RA specific military use can be augmented combat training scenarios.

\subsection{AR used in the industrial field}

Industrial augmented reality popularity is best evidenced by a survey applied DFKI German Research Institute in 2010 at the international exhibition in Hanover. This survey about potential applications of augmented reality in the industrial sector was applied to the 54 industrial representatives. The goal of the survey was to check the companies' interest in augmented reality application for industrial purposes. The results of this survey showed that $77.8 \%$ of participants saw a possible use of augmented reality for industrial purposes in the near future. Compared to the results of a survey applied in 2007 shows that the popularity of augmented reality has increased from 56\% to $85 \%$ (Schaumlöffel P., 2011).

Also from this survey resulted the industrial areas where augmented reality could be implemented and their degree of interest.

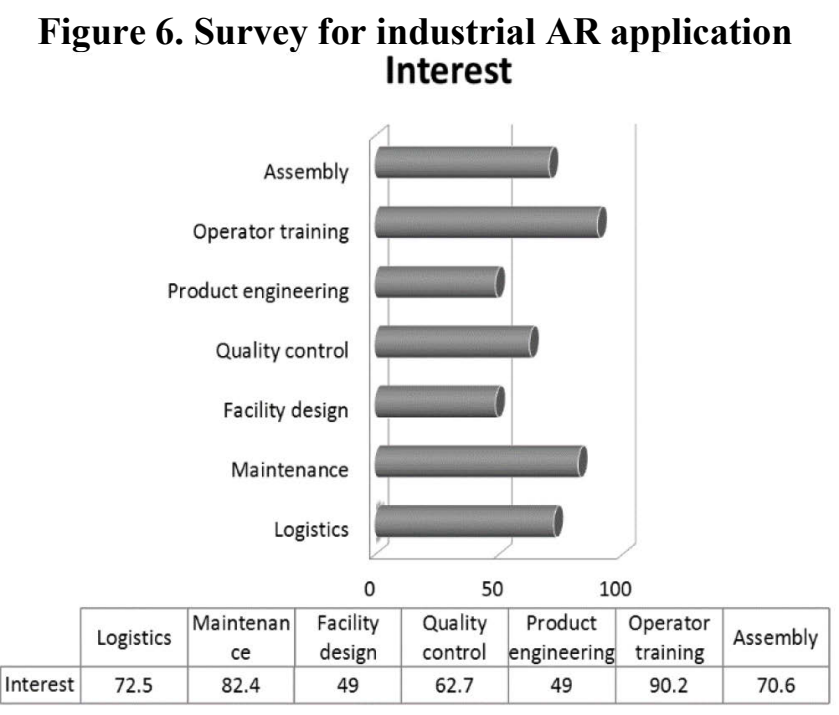

\section{Conclusions}

Computer-assisted technologies play a decisive role in shaping the environment in which we live and work. Because of this, new solutions must be found between human and machine interaction. A very important role in this context is augmented reality.

\section{References}

- Ahonen, T. (2013). Augmented Reality - the 8th Mass Medium . MongKok: TEDx.

- Bondrea I., Petruse R. (2012). Augmented Reality - An Improvement For Computer Integrated Manufacturing. International Conference on Manufacturing Engineering and Technology for Manufacturing Growth . San Diego.

- Bondrea I., Petruse R. (2011). Augmented Reality Applied in Assembly Design. 10th International MTeM Conference (pp. 41-44). Cluj-Napoca: Technical University of Cluj-Napoca

- Brîndaşu, P. D. (2008). E-learning şi e-design în domeniul sculelor aşchietoare. Sibiu.

- D.W.F. van Krevelen, R. P. (2010). A Survey of Augmented Reality Technologies, Applications and Limitations. The International Journal of Virtual Reality, 1-19.

- H. Fuchs, M. A. (1998). Augmented reality visualization for laparoscopic surgery. Proc. 1st Int'l Conf. Medical Image Computing and Computer-Assisted Intervention (pp. 934-943). Cambridge: Springer-Verlag.

- Michael R. Lyu, I. K. (n.d.). ARCADE: Augmented Reality Computing Arena for Digital Entertainment.

- Schaumlöffel P., Talha M., Gorecky D. \& Meixner G. (2011). Augmented Reality Applications For Future Manufacturing. Proceedings of the 5th Manufacturing Science and Education- MSE 2011, Vol. 1, No. 5, (June 2-5, 2011) pg. 149-152

- Xiangyu Wang, Mi Jeong Kim, Peter E.D. Love, Shih-Chung Kang (2013). Augmented Reality in built environment: Classification and implications for future research. Automation in Construction

- Xiumin Fan, R. Y. (2011). Virtual Assembly Environment for Product Design. Virtual Reality \& Augmented Reality in Industry, 147-161. 